\title{
Intravitreal Melphalan for Retinoblastoma: The Impact of Toxicity on Recurrence and Ultimate Globe Salvage
}

\author{
Jesse L. Berry ${ }^{a, b} \quad$ Mary E. Kim $^{a, b} \quad$ Maria Pefkianaki $^{a, b} \quad$ Mark Reid $^{a, b}$ \\ Rachana Shah ${ }^{c}$ Rima Jubran ${ }^{c}$ Jonathan W. Kim ${ }^{a}$ b \\ aThe Vision Center at Children's Hospital Los Angeles, Los Angeles, CA, USA; ' USC Roski Eye Institute, \\ Keck School of Medicine, University of Southern California, Los Angeles, CA, USA; ' Division of Oncology, \\ Cancer and Blood Diseases Institute, Children's Hospital Los Angeles, Keck School of Medicine, \\ University of Southern California, Los Angeles, CA, USA
}

\section{Keywords}

Retinoblastoma - Intravitreal melphalan · Toxicity ·

Recurrence · Salvage

\begin{abstract}
Introduction: Intravitreal melphalan (IVM) has emerged as an efficacious treatment for vitreous seeding in retinoblastoma. Although rarely severe, IVM-related toxicity may be treatment limiting. There is paucity of data on the impact of IVM toxicity on new tumor formation and ultimate globe salvage. Objectives: To investigate whether the grade of retinal toxicity post-IVM impacts retinal and seeding tumor recurrence, as well as the overall ability to salvage the eye. Methods: A single-institution retrospective chart review was performed on 47 eyes of 42 patients who received systemic intravenous chemotherapy followed by IVM as salvage treatment for persistent or recurrent vitreous seeding. Chorioretinal toxicity was graded from 0 to 5 . Results: Toxicity grade was inversely associated with the risk of recurrence, where a one-unit increase in toxicity grade correlated with nearly a $54 \%$ reduction in the odds of tumor recurrence (OR $0.46[0.25-0.84], p=0.01)$. Similarly, toxicity grade was related to enucleation, where a one-unit increase in toxicity grade was associated with a $31 \%$ reduction in the odds of
\end{abstract}

karger@karger.com

(c) 2020 S. Karger AG, Basel

www.karger.com/oop

Karger! undergoing enucleation (OR $0.69[0.40-1.18], p=0.17)$. Conclusions: While retinoblastoma therapy aims to limit toxicity, especially visually significant toxicity, eyes with higher grades of post-IVM toxicity are less likely to have retinal and seeding tumor recurrence.

(c) 2020 S. Karger AG, Base

\section{Introduction}

Over the past decade, there have been critical advances in the ability to salvage eyes with advanced retinoblastoma. Much of this success can be attributed to the robust efficacy in treating persistent/recurrent vitreous seeding with intravitreal injections of melphalan [1-5]. However, intravitreal melphalan (IVM) has been described to cause anterior segment toxicity [6], ERG deficits in doses $>30$ $\mu \mathrm{g}$ [7] indicative of potentially visually significant retinal toxicity, and retinal thinning on optical coherence tomography [8]. While rare, severe hemorrhagic toxicity leading to diffuse chorioretinal and optic atrophy has also been described [9]. Although severe toxicity was initially

J.L.B. and M.E.K. are co-first authors. 
thought to be associated with posterior vitreous detachment, thus concentrating the chemotherapy to the retina, a review of a larger cohort of patients failed to find an association between posterior vitreous detachment and toxicity [10]. A recent publication from a Chinese cohort described significant toxicity: $40 \%$ of patients with grade 3 or higher toxicity, $40 \%$ with vascular occlusion (similar to the acute hemorrhagic presentation), $40 \%$ anterior toxicity, and upwards of $20 \%$ with optic atrophy [11].

In retinoblastoma, the goal of cancer-directed therapy is to maximize efficacy and limit treatment-related toxicity, especially visually significant toxicity. While IVM has emerged as a safe and efficacious treatment for vitreous seeding in retinoblastoma, IVM-related toxicities can be dose or treatment limiting. The authors have noted that patients with more severe levels of post-IVM toxicity do not appear to have the same subsequent risk of recurrent retinal tumor formation, or seeding, whether in the vitreous or subretinal space. Hypothetically, this may be due to increased concentration of the chemotherapy to the retina. A similar hypothesis was made with intra-arterial chemotherapy (IAC) when it was noted that fewer new tumors form after IAC than after either systemic chemotherapy or radiation [12]. Thus, the authors aimed to retrospectively investigate whether the grade of retinal toxicity post-IVM injection impacted retinal tumor recurrence, seeding tumor recurrence, and the overall ability to salvage the eye.

\section{Materials and Methods}

Patients with newly diagnosed retinoblastoma in at least 1 eye who received systemic intravenous and/or intra-arterial chemotherapy followed by IVM as salvage treatment for persistent/recurrent vitreous seeding, from January 1, 2012, to December 31, 2018, were included in the analysis after written informed consent was obtained from the subject (parent or guardian as proxy).

\section{Chart Review}

A retrospective chart review was performed to compile the following data: age at diagnosis, gender, laterality of retinoblastoma, TNM staging, number of IVM injections, dosage of injections, interval of time between injections, toxicities, outcome after IVM, and length of follow-up.

\section{Treatment}

The protocol for chemoreduction at Children's Hospital Los Angeles (CHLA) has been published previously [13]. The dosing regimen was modified for children $<6$ months of age at the time of diagnosis [14]. At CHLA, IAC with melphalan is offered only for unilateral Group D eyes in children greater than 6 months of age [15], and IAC may also be used for relapsed disease after systemic chemotherapy. Local consolidation therapy options include diode laser therapy (532- or 810-nm laser, 1.2-mm spot size) and cryotherapy.
Patients were referred for IVM injections if either persistently active or recurrent vitreous seeds were found on examination under anesthesia (EUA). For clinically significant vitreous seeding that persisted during the course of systemic intravenous chemotherapy, we initiated IVM injections during cycles 4-6. For recurrent seeding, a course of 3 weekly injections during EUA was planned; a decision to administer or hold the injection was made each week based on the presence of seeding activity. Injections were administered until there were no signs of activity; in some patients, this resulted in fewer and in others $>3$ injections per cycle. Additional cycles of IVM were initiated for persistent seeding if seeding recurrence was observed at $\geq 4$ weeks after the completion of the previous IVM cycle. In our cohort, we did not perform an additional injection after the resolution of seeding, as has been described at other centers [16]. The dosage of IVM ranged from 20 to $40 \mu \mathrm{g}$ based on clinical features such as the age of the patient and the degree of vitreous seeding activity, which included both visible seeds and the degree of new preretinal tumors. However, once there was described toxicity at doses $>30 \mu \mathrm{g}$ in the literature $[7,17]$, a dose of $25 \mu \mathrm{g}$ was routinely used, and doses over $25 \mu \mathrm{g}$ were never given. Rarely, however, a dose of $20 \mu \mathrm{g}$ may be given for a young child under 12 months of age with very mild seeding activity, determined clinically, or who had toxicity in the other eye. The dilution of melphalan used in all cases was $20 \mu \mathrm{g} / 0.1 \mathrm{~mL}$. The IVM injection procedure for vitreous seeding in retinoblastoma eyes at CHLA closely follows the protocol outlined by Munier et al. [2] and has been published previously $[9,18]$.

\section{Staging, Toxicity Assessment, and Recurrence}

Eyes were classified according to the International Intraocular Retinoblastoma Classification (IIRC) System described by Murphree [19] and according to the AJCC TNM8 for retinoblastoma [20]. Vitreous seeding was classified into dust, spheres, or clouds based on seed morphology using the classification scheme outlined by Munier [21] and Francis et al. [4]. IVM toxicity was graded as $0-5$ for each eye using the clinical grading system described by Munier [21]. Grading of retinal toxicity was performed both by clinical assessment during EUA and retrospective review of RetCam fundus photographs. In order to carefully isolate toxicity due solely to IVM, any RPE changes from subretinal fluid at the time of diagnosis or subsequent to other therapies prior to the initiation of IVM therapy were noted in the review of records and excluded in the grading of post-IVM toxicity. Lastly, all recurrences were true scar recurrences from a previously treated tumor and not new tumor formation in the setting of heritable retinoblastoma.

\section{Images}

Images were obtained using a wide-angle contact fundus camera (RetCam; Clarity Medical Systems, Inc., Pleasanton, CA, USA) during EUA. Images for each eye included in this study were obtained at the time of diagnosis, after the first cycle of systemic chemotherapy, just before IVM therapy was initiated (within 1 month of the first injection), and just after IVM therapy was completed (within 1 month of last injection).

\section{Statistical Analysis}

We compared toxicity grade with rates of recurrence and globe salvage using logistic regression and independent samples $t$ tests. Additionally, we compared the number of IVM injections, cumulative and average melphalan dose, age at time of injection, and 
Table 1. Patient characteristics and outcomes

\begin{tabular}{|c|c|}
\hline \multicolumn{2}{|l|}{ Demographics } \\
\hline All patients included & 39 \\
\hline Number of eyes & 44 \\
\hline \multicolumn{2}{|l|}{ Gender } \\
\hline Females & $17(44)$ \\
\hline Males & $22(56)$ \\
\hline Median age at diagnosis, months & $10.7(0.4-60.2)$ \\
\hline \multicolumn{2}{|l|}{ Laterality } \\
\hline Left eye & $16(36)$ \\
\hline Right eye & $28(64)$ \\
\hline \multicolumn{2}{|l|}{ IIRC classification } \\
\hline Group A & $0(0)$ \\
\hline Group B & $3(7)$ \\
\hline Group C & $4(9)$ \\
\hline Group D & $33(75)$ \\
\hline Group E & $4(9)$ \\
\hline \multicolumn{2}{|l|}{ Seeding at time of diagnosis } \\
\hline Vitreous only & $17(39)$ \\
\hline Dust & 12 \\
\hline Sphere & 3 \\
\hline Cloud & 2 \\
\hline Subretinal only & $14(32)$ \\
\hline Vitreous and subretinal & $9(20)$ \\
\hline Dust & 6 \\
\hline Sphere & 3 \\
\hline Cloud & 0 \\
\hline None & $4(9)$ \\
\hline \multicolumn{2}{|c|}{ Quadrants of seeding (if present) at time of diagnosis } \\
\hline 1 quadrant & $5(13)$ \\
\hline 2 quadrants & $12(30)$ \\
\hline 3 quadrants & $6(15)$ \\
\hline 4 quadrants & $17(42)$ \\
\hline Median follow-up time, months & $40.33(20.7)$ \\
\hline Median time from diagnosis to IVM, months & $6.3(5.0)$ \\
\hline
\end{tabular}

toxicity grade among the 3 seed classification types using the Kruskal-Wallis test. Associations between the number of melphalan injections or total dose and IVM toxicity were evaluated using Spearman's rank-order correlations. All analyses were conducted using Stata/SE 14.2 (StataCorp LLC, College Station, TX, USA).

\section{Results}

A retrospective review was performed on patients with newly diagnosed retinoblastoma who were treated with IVM as salvage treatment for persistent or recurrent vitreous seeding from January 1, 2012, to December 31,2018 . Three eyes were excluded from the analysis because they received one or more doses of IAC, which may have confounded results. The inclusion criteria were met in 44 eyes of 39 patients. Detailed demo-

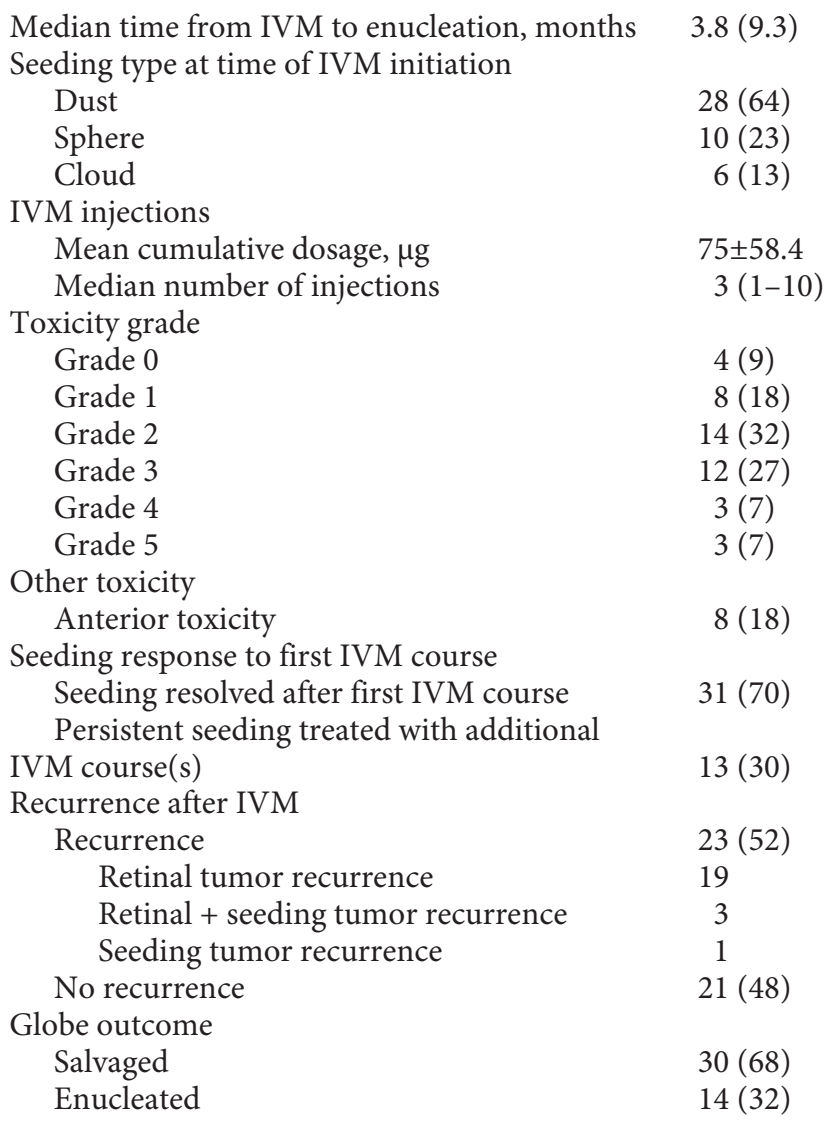

Data are presented as $n(\%)$, median (range), median (SD), or mean $\pm \mathrm{SD}$, as appropriate.

graphic information and patient characteristics are summarized in Table 1.

Of the 44 eyes included in the analysis, 28 (64\%) had a dust seeding type, $10(23 \%)$ had a sphere seeding type, and $6(13 \%)$ had a cloud seeding type. The median cumulative dosage of IVM injections received was $75 \mu \mathrm{g}$ (SD $58.4 \mu \mathrm{g})$. The total number of injections received per eye ranged from 1 to 10 , with the median being 3 injections.

Vitreous seeding resolved after the first IVM course (most often 3 injections) for 31 eyes (70\%). Therefore, these eyes received no further IVM injections and received only local treatment as necessary. Twenty-three of these eyes were ultimately salvaged ( $74 \%)$. Of note, 7 of the salvaged eyes also had a small $(<3 \mathrm{~mm})$ retinal tumor recurrence from the scar of a previously treated lesion wherein the tumor growth was effectively controlled by local consolidation laser treatment only. Eight of these 31 
Table 2. Recurrence rate for both retinal and/or subretinal seeding decreases significantly with higher grades of post-IVM toxicity

\begin{tabular}{lllll}
\hline $\begin{array}{l}\text { Toxicity } \\
\text { grade }\end{array}$ & $\begin{array}{l}\text { Recurrence, } \\
n\end{array}$ & $\begin{array}{l}\text { No } \\
\text { recurrence, } n\end{array}$ & $\begin{array}{l}\text { Total } \\
\text { eyes }\end{array}$ & $\begin{array}{l}\text { Recurrence } \\
\text { rate, } \%\end{array}$ \\
\hline 0 & 3 & 1 & 4 & 75 \\
1 & 6 & 2 & 8 & 75 \\
2 & 9 & 5 & 14 & 64 \\
3 & 4 & 8 & 12 & 33 \\
4 & 1 & 2 & 3 & 33 \\
5 & 0 & 3 & 3 & 0 \\
\hline
\end{tabular}

eyes were ultimately enucleated (26\%): 7 eyes due to retinal tumor recurrence after IVM, and 1 eye due to total retinal detachment with inability to appropriately monitor for disease recurrence.

Thirteen eyes (30\%) showed persistent seeding after the first IVM course and were consequently treated with additional IVM courses and local consolidation treatment as deemed clinically necessary. Seven of these 13 eyes (54\%) were ultimately salvaged. Five of the 7 salvaged eyes had a small $(<3 \mathrm{~mm})$ retinal tumor recurrence from a previously treated scar $(n=3)$, retinal and localized subretinal seeding tumor recurrence $(n=1)$, or solely subretinal seeding tumor recurrence $(n=1)$; these were all controlled with local laser consolidation only. The remaining 2 of the 7 salvaged eyes had no subsequent recurrence following the completion of additional IVM courses, resulting in no further need for systemic treatment or for local consolidation therapy. Six of the 13 eyes (46\%) were ultimately enucleated. All cases of enucleation were due to tumor recurrence after IVM, with 4 eyes displaying solely retinal tumor recurrence and 2 eyes displaying both retinal and seeding tumor recurrence.

One patient in our study died of metastatic disease that developed during the median follow-up period of 39.6 months (SD 20.9 months). This patient was found to have high-risk histopathology upon secondary enucleation and was treated with 6 cycles of adjuvant chemotherapy per Children's Oncology Group (COG) protocol ARET0332. He developed metastatic disease 13 months after treatment.

The number of eyes that displayed each grade of toxicity upon conclusion of treatment can be found in Table 1 .

\section{IVM Toxicity Relationship with Recurrence and \\ Enucleation Rates}

IVM toxicities were observed in 44 eyes: grade 0 ( $n=$ $4), 1(n=8), 2(n=14), 3(n=12), 4(n=3)$, and $5(n=$
Table 3. IVM toxicity relationship with recurrence and enucleation rates

$\begin{array}{ll}\text { IVM toxicity and recurrence } & \\ \text { Odds ratio } & 0.46 \\ \text { CI } & 0.25-0.84 \\ p \text { value } & 0.012 \\ \text { IVM toxicity and enucleation } & \\ \quad \text { Odds ratio } & 0.69 \\ \text { CI } & 0.40-1.18 \\ p \text { value } & 0.14\end{array}$

Table 4. Injection number and dose by seed class

\begin{tabular}{lll}
\hline & Injections, $n$ & $\begin{array}{l}\text { Cumulative melphalan } \\
\text { dosage, } \mu \mathrm{g}\end{array}$ \\
\hline Dust & $3.1 \pm 1.4$ & $80.0 \pm 39.1$ \\
Sphere & $4.3 \pm 1.7$ & $111.0 \pm 55.4$ \\
Cloud & $5.7 \pm 3.0$ & $159.4 \pm 94.4$ \\
\hline
\end{tabular}

Data are presented as mean $\pm \mathrm{SD}$.

3). Anterior toxicity was observed in 8 eyes. Toxicity grade was significantly associated with recurrence, where a one-unit increase in toxicity grade correlated with nearly a $54 \%$ reduction in the odds of tumor recurrence (OR $0.46[0.25-0.84])$. For example, an eye with grade 2 toxicity was $54 \%$ less likely to display tumor recurrence than an eye with grade 1 toxicity, and this relationship held for each unit increase in toxicity. Expressed another way, the average IVM toxicity grade of eyes showing recurrence (1.74, averaged toxicity grade) was lower than that for eyes that did not show recurrence $(2.81, p=0.005$; Table $2)$. Similarly, toxicity grade was related to enucleation, where a one-unit increase in toxicity grade was associated with a $31 \%$ reduction in the odds of undergoing enucleation (OR 0.69 [0.40-1.18]; Table 3).

\section{Seed Class Is Not a Predictor of IVM Toxicity}

As shown previously [3], the total number of IVM injections was significantly associated with seed class $(p=$ 0.02 ). Eyes with cloud seed morphology received significantly more injections, specifically ranging from 3.1 (dust) to 5.7 (cloud). Additionally, the median total cumulative dosage of melphalan increased from 80.0 (dust) to 159.4 (cloud), a statistically significant difference ( $p=$ 0.04 ; Table 4). 
Fig. 1. Images taken at time of diagnosis and after completion of IVM treatment. OD: Group C with dust seeding at diagnosis (a); grade 4 toxicity post-IVM (b). OS: Group D with sphere seeding at diagnosis (c); grade 1 toxicity post-IVM (toxicity in periphery only) (d). The left eye was ultimately enucleated due to retinal tumor recurrence under the calcium of the tumor along the inferior arcade.

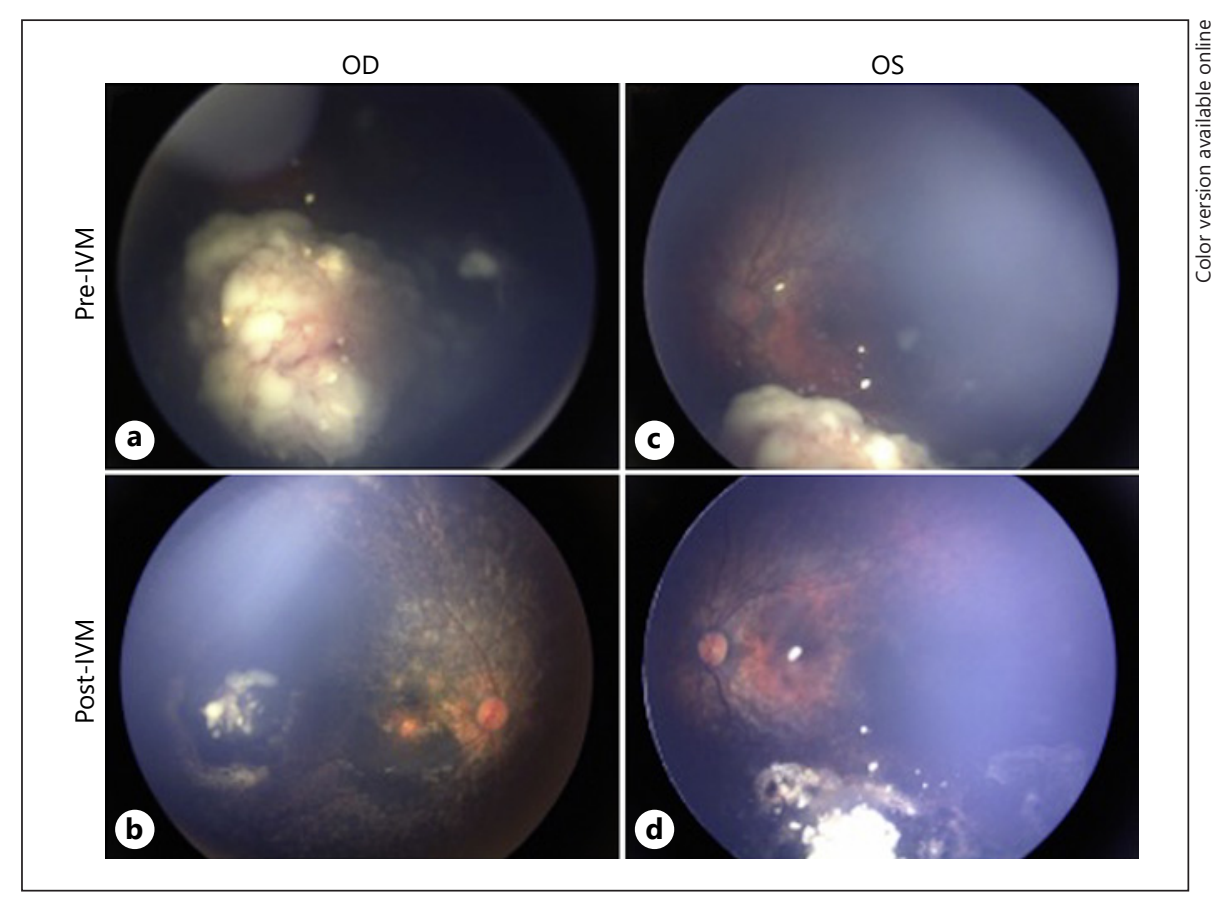

While the overall dose and number of injections correlated positively with seed class, both correlated negatively with toxicity ( $\rho=-0.36$ and $p=0.01$ for dose, $\rho=$ -0.48 and $p=0.001$ for number of injections). This would suggest that eyes receiving fewer injections and a lower cumulative dose of melphalan are less likely to develop toxicity; however, this is confounded by the fact that injections were stopped immediately when higher-grade toxicity was noted. Eyes with grade 4 or 5 toxicity received a median of 2 injections (range 1-3). Because the number of injections and total dose measure the same construct (overall melphalan doses), and the relationship between doses and toxicity grade is confounded by standard clinical protocols (e.g., where eyes with grades 4 and 5 are not given any further injections), we did not conduct additional multivariate analyses as these would also be confounded by standard clinical protocols. Seed class was not associated with higher levels of toxicity $(p=0.48)$.

\section{Discussion}

In this study, the grade of chorioretinal toxicity after IVM was inversely associated with risk of tumor recurrence. Eyes with higher grades of toxicity demonstrated lower rates of subsequent retinal and seeding tumor recurrence, and thus decreased consolidation requirements post-IVM and improved overall success in globe salvage.
Our results demonstrate that a one-unit increase in toxicity grade correlated with nearly a 55\% reduction in the odds of a post-IVM recurrence. This may imply that the retina (and thus retinal tumors) in eyes with higher toxicity grades are acutely exposed to a higher concentration of melphalan compared to eyes that receive comparable cumulative melphalan dosages but display lower toxicity grades [21]. A similar hypothesis has been proposed for eyes treated with IAC [12]; however, whether this is due to anatomic, metabolic, or other variations in the setting of IVM is unclear. While this higher concentration of chemotherapy manifests as toxicity, it also functions to reduce both retinal and seeding tumor recurrence. Therefore, although IVM has traditionally been employed as a treatment for vitreous seeding [1-5], it appears to demonstrate some efficacy in preventing recurrent retinal tumor growth as well as subretinal seeding [22, 23]. It is important to note that treatment schemes in advanced retinoblastoma eyes are multimodal; additional local consolidation therapy including laser and/or cryotherapy was required by the eyes included in this review, and this local therapy aided in tumor resolution and ultimate globe salvage. This consolidation therapy may have contributed to the significant inverse relationship seen between toxicity grade and recurrence rate as a confounding variable.

Consistent with previously published reports [3], our data suggest that none of the following was predic- 
tive of higher levels of toxicity: increased number of injections, increased cumulative dosage of melphalan, or seed class. However, higher levels of toxicity were predictive of a decreased risk for further retinal and/or vitreous tumor recurrences in this retrospective evaluation of eyes treated with systemic chemoreduction and intravitreal injection of melphalan. While singular IVM dosages higher than $30 \mu \mathrm{g}$ may increase the risk of toxicity, neither the cumulative dosage nor the number of injections that an eye received correlates with toxicity grade [17]. It has also been previously suggested that more deeply pigmented eyes may be at increased risk for toxicity due to increased absorption of melphalan by the choroid and retinal pigment epithelium [7, 11, 24, $25]$. Regardless, not all deeply pigmented eyes experience IVM toxicity. Thus, there is presently no definitive way to predict whether toxicity, specifically higher grades of toxicity, will occur or not. It should be noted that, as in clinical practice, eyes with grade 4 or 5 toxicity were a small portion of our sample size, which is a limitation of this study.

A case example may be seen in one of our Hispanic patients with bilateral retinoblastoma and seeding treated initially with systemic chemotherapy followed by intravitreal chemotherapy. The right eye of the patient was diagnosed as Group C with dust seeding, while the left eye was diagnosed as Group D with sphere seeding. After 6 cycles of intravenous chemotherapy, the seeding persisted bilaterally. Therefore, the right eye received $60 \mu \mathrm{g}$ of cumulative melphalan, and demonstrated grade 4 toxicity involving the macula after the third injection. Given the toxicity, no further injections were given. However, no subsequent retinal or seeding recurrences were seen and no further local consolidation treatment was required. In contrast, the patient's left eye received $65 \mu \mathrm{g}$ of intravitreal chemotherapy over 3 injections. The eye demonstrated grade 1 toxicity, and both the retinal recurrence and persistent seeding responded minimally to this therapy. The left eye was ultimately enucleated after local therapy failed to control the intraocular disease (Fig. 1). There are multiple confounding variables here aside from the level of post-IVM toxicity, most notably the fact that the enucleated left eye was more advanced at diagnosis and thus always at an increased risk of requiring enucleation. Nonetheless, it is notable that 2 eyes of the same patient, both with persistent seeding after 6 cycles of intravenous chemotherapy, were subjected to a similar overall dosage of chemotherapy and yet developed a range of toxicity 3 grades apart. Additionally, a lack of all subsequent tumor activity in the right eye after grade 4

The Risk of Recurrence after Intravitreal Melphalan Toxicity toxicity was observed, as opposed to continued recurrences in the left, illustrating the common disease course that was observed in eyes after high grades of toxicity were seen.

In clinical practice, this finding does not suggest that clinicians should aim for higher grades of toxicity, but rather that eyes with higher grades of toxicity may be at a lower risk of tumor recurrence and may require fewer subsequent sessions of local consolidation for ocular salvage. Unfortunately, this tumoricidal benefit has a significant cost as visual potential is often quite limited for eyes with higher grades (4/5) of toxicity. Further, while we understand some risk factors, the mechanism of severe toxicity after IVM is not yet clear. Herein we present real-world data from a large retinoblastoma center and share observations and associations regarding the toxicity of this treatment. As chemotherapy regimens evolve, these observations will be critical for implementing the best care for these patients. For example, while by no means definitive, the findings presented herein lend support to the fact that IVM may play a role in treating small retinal tumors and, as with IAC, in preventing new or recurrent retinal and subretinal disease in addition to treating vitreous seeds. Taken together, this information can aid the ocular oncologist in best applying consolidative therapies during attempts at globe salvage, specifically in the setting of post-IVM toxicity, for patients with retinoblastoma.

\section{Statement of Ethics}

This study was approved by the Institutional Review board at CHLA (IRB No. CHLA-17-00248) and conformed to the requirements of the US Health Insurance Portability and Privacy Act. The research was conducted ethically in accordance with the World Medical Association Declaration of Helsinki. Written informed consent was obtained from all subjects (parent or guardian as proxy).

\section{Conflict of Interest Statement}

The authors have no conflicts of interest to declare.

\section{Funding Sources}

In kind, financial support was provided by: The Larry and Celia Moh Foundation; The Institute for Families, Inc., CHLA; and an unrestricted departmental grant from Research to Prevent Blindness. 
Dr. Jesse L. Berry holds grants not directly related to the support of this work from: National Cancer Institute of the National Institute of Health (Award No. K08CA232344); Hyundai Hope on Wheels; and Childhood Eye Cancer Trust.

The sponsors had no role in the study design; the collection, analysis, and interpretation of data; the writing of the report; or the decision to submit the article for publication.

\section{Author Contributions}

J.L.B., J.W.K., R.S., and R.J. conceived of the presented idea. M.E.K. and M.P. carried out the data collection and data analysis. M.R. helped perform the computations and verify the analytical methods. All authors discussed the results and contributed to the final manuscript.

\section{References}

1 Munier FL, Gaillard MC, Balmer A, Soliman S, Podilsky G, Moulin AP, et al. Intravitreal chemotherapy for vitreous disease in retinoblastoma revisited: from prohibition to conditional indications. Br J Ophthalmol. 2012 Aug;96(8):1078-83.

2 Munier FL, Soliman S, Moulin AP, Gaillard MC, Balmer A, Beck-Popovic M. Profiling safety of intravitreal injections for retinoblastoma using an anti-reflux procedure and sterilisation of the needle track. Br J Ophthalmol. 2012 Aug;96(8):1084-7.

3 Berry JL, Bechtold M, Shah S, Zolfaghari E, Reid M, Jubran R, et al. Not All Seeds Are Created Equal: Seed Classification Is Predictive of Outcomes in Retinoblastoma. Ophthalmology. 2017 Dec;124(12):1817-25.

4 Francis $\mathrm{JH}$, Abramson DH, Gaillard MC, Marr BP, Beck-Popovic M, Munier FL. The classification of vitreous seeds in retinoblastoma and response to intravitreal melphalan. Ophthalmology. 2015 Jun;122(6):1173-9.

5 Berry JL, Shah S, Bechtold M, Zolfaghari E, Jubran R, Kim JW. Long-term outcomes of Group D retinoblastoma eyes during the intravitreal melphalan era. Pediatr Blood Cancer. 2017 Dec;64(12):e26696.

6 Francis JH, Marr BP, Brodie SE, Abramson DH. Anterior Ocular Toxicity of Intravitreous Melphalan for Retinoblastoma. JAMA Ophthalmol. 2015 Dec;133(12):1459-63.

7 Francis JH, Schaiquevich P, Buitrago E, Del Sole MJ, Zapata G, Croxatto JO, et al. Local and systemic toxicity of intravitreal melphalan for vitreous seeding in retinoblastoma: a preclinical and clinical study. Ophthalmology. 2014 Sep;121(9):1810-7.

8 Narala R, Kim JW, Lang P, Le BH, Hendargo $\mathrm{HC}$, Branco D, et al. Changes in Retinal Thickness on OCT from Intravitreal Melphalan. Ophthalmol Retina. 2019 Mar;3(3):2889.
9 Aziz HA, Kim JW, Munier FL, Berry JL. Acute hemorrhagic retinopathy following intravitreal melphalan injection for retinoblastoma: a report of two cases and technical modifications to enhance the prevention of retinal toxicity. Ocul Oncol Pathol. 2017 Jan;3(1):34-40.

10 Berry JL, Lee R, Patel L, Le BH, O'Fee J, Jubran $\mathrm{R}$, et al. Posterior Vitreous Detachment and the Associated Risk of Retinal Toxicity with Intravitreal Melphalan Treatment for Retinoblastoma. Ocul Oncol Pathol. 2019 Jun;5(4): 238-44.

11 Xue K, Ren H, Meng F, Zhang R, Qian J. Ocular toxicity of intravitreal melphalan for retinoblastoma in Chinese patients. BMC Ophthalmol. 2019 Feb;19(1):61.

12 Abramson DH, Francis JH, Dunkel IJ, Marr BP, Brodie SE, Gobin YP. Ophthalmic artery chemosurgery for retinoblastoma prevents new intraocular tumors. Ophthalmology. 2013 Mar; 120(3):560-5.

13 Berry JL, Jubran R, Kim JW, Wong K, Bababeygy SR, Almarzouki $H$, et al. Long-term outcomes of Group D eyes in bilateral retinoblastoma patients treated with chemoreduction and low-dose IMRT salvage. Pediatr Blood Cancer. 2013 Apr;60(4):688-93.

14 Berry JL, Jubran R, Lee TC, Murphree AL, Lee D, Kim JW. Low-dose chemoreduction for infants diagnosed with retinoblastoma before 6 months of age. Ocul Oncol Pathol. 2015 Feb; $1(2): 103-10$.

15 Manjandavida FP, Stathopoulos C, Zhang J, Honavar SG, Shields CL. Intra-arterial chemotherapy in retinoblastoma - A paradigm change. Indian J Ophthalmol. 2019 Jun;67(6): $740-54$.
16 Shields CL, Douglass AM, Beggache M, Say EA, Shields JA. Intravitreous chemotherapy for active vitreous seeding from retinoblastoma. Retina. 2016;36(6):1184-90.

17 Smith SJ, Smith BD, Mohney BG. Ocular side effects following intravitreal injection therapy for retinoblastoma: a systematic review. $\mathrm{Br} \mathrm{J}$ Ophthalmol. 2014 Mar;98(3):292-7.

18 Berry JL, Bechtold M, Shah S, Zolfaghari E, Reid M, Jubran R, et al. Not all seeds are created equal: seed classification is predictive of outcomes in retinoblastoma. Ophthalmology. 2017 Dec;124(12):1817-25.

19 Linn Murphree A. Intraocular retinoblastoma: the case for a new group classification. Ophthalmol Clin North Am. 2005 Mar;18(1): 41-53.

20 TNM8. TNM8: the updated TNM classification for retinoblastoma. Community Eye Health. 2018;31(101):34.

21 Munier FL. Classification and management of seeds in retinoblastoma. Ellsworth Lecture Ghent August 24th 2013. Ophthalmic Genet. 2014 Dec;35(4):193-207.

22 Abramson DH, Ji X, Francis JH, Catalanotti F, Brodie SE, Habib L. Intravitreal chemotherapy in retinoblastoma: expanded use beyond intravitreal seeds. Br J Ophthalmol. 2019 Apr;103(4):488-93.

23 Abramson DH, Catalanotti F, Brodie SE, Kellick MG, Francis JH. Intravitreal chemotherapy and laser for newly visible subretinal seeds in retinoblastoma. Ophthalmic Genet. 2018 Jun;39(3):353-6.

24 Francis JH, Brodie SE, Marr B, Zabor EC, Mondesire-Crump I, Abramson DH. Efficacy and toxicity of intravitreous chemotherapy for retinoblastoma: four-year experience. Ophthalmology. 2017 Apr;124(4):488-95.

25 Singh AD. Retinoblastoma: imaging, lasers, and injections. Ophthalmol Retina. 2017 SepOct;1(5):359-60. 